M. Itô

Nagoya Math. J.

Vol. 37 (1970), 81-90

\title{
SUR LES FONGTIONS POLYHARMONIQUES ET LE PROBLEME DE RIQUIER
}

\author{
En hommage à Monsieur le professeur Katuji ONO á \\ l'occasion de sa 60ème année
}

\section{MASAYUKI ITO}

\section{Introduction}

Soit $\Omega$ un ouvert de l'espace euclidien $R^{n}$ à $n(\geqq 2)$ dimensions. Les recherches des fonctions harmoniques dans $\Omega$ sont essentielles dans la théorie classique du potentiel. A ce moment-là, il est bien connu que le balayage pour le noyau de Green dans $\Omega$ joue un grand rôle (par example, cf. $\mathrm{M}$. Brelot [1]).

D'autre part, quelques travaux ont été consacrés à l'étude de la fonction polyharmonique dans $\Omega$, et on sait maintenant que les résultats sont souvent pareils à la théorie des fonctions harmoniques dans $\Omega$ (cf. M. Nicolesco [3]). Mais il était nécessaire de faire quelques restrictions, car on ne connaissait pas de balayage pour le noyau polypotentiel.

Nous considérerons ici le balayage pour le noyau polypotentiel dans $\Omega$, qui sera très différent du balayage ordinaire. Il en résultera immédiatement le théorème de valeur moyenne pour la fonction polyharmonique dans $\Omega$, et puis nous donnerons la solution explicite du problème de Riquier pour l'équation polyharmonique $\Delta^{p} u=0$ et pour un ouvert borné de $R^{n}$. Il sera caractéristique de voir que les points irréguliers seront les mêmes que pour l'équation de Laplace. Cette étude précise la note [2].

\section{Le noyau polypotentiel et le balayage}

Dans cette section, on supposera toujours que $X$ sera un ouvert borné de $R^{n}$ à $n \geqq 2$. On pose, pour un entier $p \geqq 1$,

$$
N^{(p)}(x, y)=\int \cdots \int G\left(x, z_{1}\right) G\left(z_{1}, z_{2}\right) \cdots G\left(z_{p-1}, y\right) d z_{1} \cdots d z_{p-1},
$$

Received December 17, 1968 
où $G$ est la fonction de Green dans $X$. La fonction $N^{(p)}$ est positive, symétrique et continue au sens large dans $X \times X$; elle est finie et continue dans $X \times X-\delta$, où $\delta$ est la diagonale de $X \times X$. Si $G$ est convenablement normalisée, on a

$$
\left(\Delta_{y}\right)^{p} N^{(p)}(x, y)=(-1)^{p} \varepsilon_{x}
$$

au sens des distributions dans $\Omega$, où $\varepsilon_{x}$ est la mesure de Dirac à $x$. Pour une mesure de Radon positive $\mu$ dans $X$, on note $N^{(p)} \mu(x)$ la valeur en $x$ $\mathrm{du}$ potentiel de $\mu$ par rapport au noyau $N^{(p)}$. Les fonctions $N^{(p)}(x, y)$ et $N^{(p)} \mu(x)$ s'appelle respectivement le noyau polypotentiel d'ordre $p$ et le potentiel d'ordre $p$ de $\mu$.

ThÉorème 1 (le théorème du balayage): Soit $p \geqq 1$. $A$ un système $\left(\mu_{i}\right)_{i=1}^{p}$ de mesures de Radon positives dans $X$, de masse totale finie, et à un fermé $F$ de $X$, on peut associer un système $\left(\mu_{i}^{\prime}\right)_{i=1}^{p}$ de mesures positives portée par $F$, et un seul tel qu'on ait

$$
\begin{aligned}
& \sum_{i=1}^{p} N^{(p-i+1)} \mu_{i}(x) \geqq \sum_{i=1}^{p} N^{(p-i+1)} \mu_{i}^{\prime}(x) \quad \text { partout dans } X, \\
& \sum_{i=1}^{p} N^{(p-i+1)} \mu_{i}(x)=\sum_{i=1}^{p} N^{(p-i+1)} \mu_{i}^{\prime}(x) \quad \text { quasi partout sur } F, \\
& \sum_{i=1}^{p-1} N^{(p-i)} \mu_{i}(x)=\sum_{i=1}^{p-1} N^{(p-i)} \mu_{i}^{\prime}(x) \quad \text { quasi partout sur } F, \\
& \ldots \ldots \ldots \ldots \ldots \ldots \ldots \ldots \\
& N^{(1)} \mu_{1}(x)=N^{(1)} \mu_{1}^{\prime}(x) \text { quasi partout sur } F .
\end{aligned}
$$

On dit qu'une propriété a lieu quasi partout si elle a lieu sauf sur un ensemble de capacité extérieure nulle pour le noyau de Green ou ce qui revient au même, pour le noyau newtonnien (logarithmique pour $n=2$ ).

Démonstration. Nous remarquons d'abord que le potentiel d'ordre 1 de $\mu_{i}$ est finie quasi partout dans $X$ et l'on a $\int N^{(1)} \mu_{i}(x) d x<+\infty$. Donc, pour $1 \leqq i \leqq p$ et pour $2 \leqq j \leqq p, N^{(j)} \mu_{i}$ est finie quasi partout dans $X$.

Soit $\mu_{1}^{\prime}$ la mesure balayée de $\mu_{1}$ sur $F$ relativement au noyau $G$, on obtient alors

$$
\begin{array}{ll}
N^{(1)} \mu_{1}(x) \geqq N^{(1)} \mu_{1}^{\prime}(x) & \text { partout dans } X, \\
N^{(1)} \mu_{1}(x)=N^{(1)} \mu_{1}^{\prime}(x) & \text { quasi partout sur } F .
\end{array}
$$


Ensuite, soit $\mu_{2}^{\prime}$ la mesure balayée de $\left(N^{(1)} \mu_{1}-N^{(1)} \mu_{1}^{\prime}\right)+\mu_{2}$ relativement à $G$. Ayant

$$
N^{(2)} \mu_{1}(x)-N^{(2)} \mu_{1}^{\prime}(x)+N^{(1)} \mu_{2}(x)=\int G(x, y)\left(\left(N^{(1)} \mu_{1}(y)-N^{(1)} \mu_{1}^{\prime}(y)\right) d y+d \mu_{2}(y)\right),
$$

on a

$$
\begin{array}{ll}
N^{(2)} \mu_{1}(x)+N^{(2)} \mu_{2}(x) \geqq N^{(2)} \mu_{1}^{\prime}(x)+N^{(1)} \mu_{2}^{\prime}(x) & \text { partout dans } X, \\
N^{(2)} \mu_{1}(x)+N^{(1)} \mu_{2}(x)=N^{(2)} \mu_{1}^{\prime}(x)+N^{(1)} \mu_{2}^{\prime}(x) & \text { quasi partout sur } F .
\end{array}
$$

En répétant cette action, on obtient un système $\left(\mu_{i}^{\prime}\right)_{i=1}^{p}$ de mesures positives portées par $F$ qui vérifie tous les conditions ci-dessus.

Finalement nous montrons l'unicité de ce système. Soit $\left(\mu_{i}^{\prime \prime}\right)_{i=1}^{p}$ un autre système qui vérifie les conditions ci-dessus. On a évidemment $\mu_{1}^{\prime}=\mu_{1}^{\prime \prime}$, et ensuite on a

$$
\begin{aligned}
& N^{(2)} \mu_{1}(x)-N^{(2)} \mu_{1}^{\prime}(x)+N^{(1)} \mu_{2}(x)=\int G(x, y)\left(\left(N^{(1)} \mu_{1}(y)-N^{(1)} \mu_{1}^{\prime}(y)\right) d y+d \mu_{2}(y)\right) \\
& =N^{(1)} \mu_{2}^{\prime \prime}(x) \quad \text { quasi partout sur } F .
\end{aligned}
$$

Par conséquent, $\mu_{2}^{\prime \prime}$ doit être la mesure balayée de $\left(N^{(1)} \mu_{1}-N^{(1)} \mu_{1}^{\prime}\right)+\mu_{2}$ sur $F$ relativement à $G$, d'où $\mu_{2}^{\prime}=\mu_{2}^{\prime \prime}$. De la même manière, on obtient l'unicité. La démonstration est ainsi complète.

On dit que $\left(\mu_{i}^{\prime}\right)_{i=1}^{p}$ est le système balayé de $\left(\mu_{i}\right)_{i=1}^{p}$ sur $F$ relativement au système $\left(N^{(j)}\right)_{j=1}^{p}$. Il est facile de voir que si l'on a $\mu_{i}(F)=0$ pour tout $i, \mu_{i}^{\prime}$ est alors portée par la frontière $F^{*}$ de $F$. Par la suite on désignera $\operatorname{par}\left(\varepsilon_{x, F}^{(i)}\right)_{i=1}^{p}$ le système balayé de $\left(\varepsilon_{x}, 0, \cdots, 0\right)$ sur $F$.

Proposition 1. Soient $X_{1}$ et $X_{2}$ deux ouverts bornés de $R^{n}$, et soient $N_{1}^{(i)}$ et $N_{2}^{(i)}$ respectivement les noyaux polypotentiels d'ordre $i$ dans $X_{1}$ et dans $X_{2}$. Pour un système $\left(\mu_{i}\right)_{i=1}^{p}$ de mesures de Radon positives à support compact dans $X_{1} \cap X_{2}$ et pour un fermé $F$ dont le complément $C F$ est borné dans $X_{1} \cap X_{2}$, on a $\mu_{i, 1}^{\prime}=\mu_{i, 2}^{\prime}$ pour tout $1 \leqq i \leqq p$, où $\left(\mu_{i, 1}^{\prime}\right)_{i=1}^{p}$ et $\left(\mu_{i, 2}^{\prime}\right)_{i=1}^{p}$ sont respectivement les systèmes balavés de $\left(\mu_{i}\right)_{i=1}^{p}$ sur $F$ relativement $\grave{a}\left(N_{1}^{(i)}\right)_{i=1}^{p}$ et $\grave{a}\left(N_{2}^{(i)}\right)_{i=1}^{p}$.

En effet, soit $N_{1,2}^{(i)}$ le noyau polypotentiel d'ordre $i$ dans $X_{1} \cap X_{2}$, et soit $\left(\nu_{i}^{\prime}\right)_{i=1}^{p}$ le système balayé de $\left(\mu_{i}\right)_{i=1}^{p}$ sur $F$ relativement à $\left(N_{1,2}^{(i)}\right)_{i=1}^{p}$. Il est facile de voir que $\mu_{i, 1}^{\prime}=\nu_{i}^{\prime}$ et que $\mu_{i, 2}^{\prime}=\nu_{i}^{\prime}$.

Proposition 2. Soit e un sous-ensemble borélien de $F$. La fonction $f_{i}(x)=$ $\varepsilon_{x, F}^{(i)}(e)$ est analytique dans $C F$ pour tout $i$. 
En effet, il est bien connu que $f_{1}$ est analytique dans $C F$ (cf. M. Brelot [1]). D'après l'égalité

$$
f_{2}(x)=\int\left(G(x, y)-G(x, z) d \varepsilon_{y, F}^{(1)}(z)\right) \varepsilon_{y, F}^{(1)}(e) d y,
$$

il est facile de montrer que $f_{2}$ est analytique dans $C F$. Au moyen de l'induction, on peut montrer que $f_{i}$ est analytique dans $C F$ pour tout $i$.

Soit $\Omega$ un ouvert dans $X$. On désigne par $N_{\Omega}^{(p)}$ le noyau polypotentiel d'ordre $p$ dans $\Omega$. Alors on obtient la proposition suivante:

Proposition 3. Pour tous les points $x$ et $y$ de $\Omega$, on a

$$
N_{\Omega}^{(p)}(x, y)=N^{(p)}(x, y)-\sum_{i=1}^{p} N^{(p-i+1)} \varepsilon_{x, C \Omega}^{(i)}(y)
$$

En effet, le cas de $p=1$ est bien connu. Supposons que

$$
N_{\Omega}^{(p-1)}(x, y)=N^{(p-1)}(x, y)-\sum_{i=1}^{p-1} N^{(p-i)} \varepsilon_{x, C \Omega}^{(i)}(y)
$$

dans $\Omega \times \Omega$. Alors on obtient, d'après le théorème 1 , que, pour tous les points $x$ et $y$ de $\Omega$,

$$
\begin{aligned}
& N^{(p)}(x, y)-\sum_{i=1}^{p} N^{(p-i+1)} \varepsilon_{x, C \Omega}^{(1)}(y) \\
& =\int G(y, z) N_{\Omega}^{(p-1)}(z, x) d z-\int G(y, z) d \varepsilon_{x, C \Omega}^{(p)}(z) \\
& =\int G(y, z) N_{\Omega}^{(p)}(z, x) d z-\iint G(y, u) d \varepsilon_{z, C \Omega}^{(1)}(u) N_{\Omega}^{(p-1)}(z, x) d z \\
& =\int G_{\Omega}(y, z) N^{(p-1)}(z, x) d z=N_{\Omega}^{(p)}(x, y) .
\end{aligned}
$$

D'après l'induction, la démonstration est ainsi complète.

De la proposition ci-dessus, on obtient immédiatement les corollaires suivants:

Corollaire 1. La fonction $\sum_{i=1}^{p} N^{(p-j+1)} \varepsilon_{x, C \Omega}^{(i)}(y)$ dans $\Omega \times \Omega$ est symétrique.

Corollaire 2. Pour un point $x$ fixé de $\Omega$, on a

$$
\left(\Delta_{y}\right)^{p}\left(\sum_{i=1}^{p} N^{(p-i+1)} \varepsilon_{y, C \Omega}^{(i)}(x)\right)=0
$$


au sens des distributions dans $\Omega$.

Proposition 4. Soit $\mu$ une mesure de Radon positive dans $X$ de masse totale finie, et soit $F$ un fermé de $X$ avec $S_{\mu} \cap F=\phi$, alors on a, pour tout ensemble borélien $e$ de $F$ et pour tout $i$,

$$
\mu_{i}^{\prime}(e)=\int \varepsilon_{x, F}^{(i)}(e) d \mu(x)
$$

où $\left(\mu_{i}^{\prime}\right)_{i=1}^{p}$ est le système balayé de $(\mu, 0, \cdots, 0)$ sur $F$.

En effet, le cas de $i=1$ est bien connu (cf. M. Riesz [4]). Soit $i>1$ et supposons que, pour tout $j \leqq i-1, \mu_{j}^{\prime}(e)=\int \varepsilon_{x, F}^{(j)}(e) d \mu(x)$. On a alors

$$
\begin{aligned}
& \mu_{i}^{\prime}(e)=\int\left(N^{(i-1)} \mu(x)-\sum_{j=1}^{i-1} N^{(i-j)} \mu_{j}^{\prime}(x)\right) \varepsilon_{x, F}^{(1)}(e) d x \\
& =\iint\left(N^{(i-1)}(x, y)-\sum_{j=1}^{i-1} N^{(i-j)} \varepsilon_{y, F}^{(j)}(x)\right) d \mu(y) \varepsilon_{x, F}^{(1)}(e) d x \\
& =\iint\left(N^{(i-1)}(x, y)-\sum_{j=1}^{i-1} N^{(i-j)} \varepsilon_{y, F}^{(j)}(x)\right) \varepsilon_{x, F}^{(1)}(e) d x d \mu(y) \\
& =\int \varepsilon_{y, F}^{(i)}(e) d \mu(y) .
\end{aligned}
$$

D'après cette proposition, on a évidemment l'inégalité suivante: Pour tout $x$ de $X$ et pour tout fermé $F$ de $X$,

$$
N^{(p)} \mu(x) \geqq \sum_{i=1}^{p} \int N^{(p-i+1)} \mu(y) d \varepsilon_{x, F}^{(i)}(y)
$$

où $\mu$ est une mesure positive de masse totale finie.

\section{Les fonctions polyharmoniques}

Soit $\Omega$ un ouvert de $R^{n}$. Rappelons qu'une fonction réelle $u$ de classe $C^{2(p-1)}$ dans $\Omega$ est polyharmonique d'ordre $p$ si l'on a $\Delta^{p} u=0$ dans $\Omega$ au sens des distributions. Considérons le théorème de valeur moyenne pour le fonction polyharmonique dans $\Omega$. Nous donnerons d'abord une notation. On désignera par $\left(\varepsilon_{x, r}^{(i)}\right)_{l=1}^{p}$ le système balayé de $\left(\varepsilon_{x}, 0, \cdots, 0\right)$ sur $C B(x ; r)$ relativement au système de noyaux polypotentiels dans un ouvert borné $X \supset \overline{B(x ; r)}$, où $B(x ; r)$ est la boule ouverte de centre $x$ et de rayon $r$. D'après la proposition $1,\left(\varepsilon_{x, r}^{(i)}\right)_{i=1}^{p}$ est indépendant de $X$. 
Théorème 2. Pour qu'une fonction réelle $u$ de classe $C^{2(p-1)}$ dans $\Omega$ soit polyharmonique d'ordre $p$ dans $\Omega$, il faut et il suffit que, pour toute boule ouverte $B(x ; r)$ avec $\overline{B(x ; r)} \subset \Omega$, on ait

$$
u(x)=\sum_{i=1}^{p} \int(-\Delta)^{i-1} u(y) d \varepsilon_{x, r}^{(i)}(y)
$$

où $\Delta^{0} u=u$.

Démonstration de la condition suffisante. Nous remarquons d'abord que, pour tous les points $x$ et $y$ de $\Omega$ et pour tout $i, \varepsilon_{y, r}^{(i)}$ est la mesure obtenue par la translation $y-x$ de $\varepsilon_{x, r}^{(i)}$, et que par suite, il suffit de montrer le lemme suivant:

Lemme 1. Pour tout $x$ de $R^{n}$ et pour toute suite décroissante $\left(r_{m}\right)$ de nombres $>0$ s'annulant avec $m \rightarrow \infty$, il existe une suite $\left(a_{m}\right)$ de nombres $>0$ avec $\lim _{m \rightarrow \infty} a_{m}=0$ telle qu'on ait, pour toute fonction $\varphi$ de classe $C^{2 p}$ à support compact dans $R^{n}$,

$$
(-\Delta)^{p} \varphi(x)=\lim _{m \rightarrow \infty} \frac{1}{a_{m}}\left(\varphi(x)-\sum_{i=1}^{p} \int(-\Delta)^{i-1} \varphi(y) d \varepsilon_{x, r_{m}}^{(i)}(y)\right) .
$$

En effet, soit $X$ un ouvert borné de $R^{n}$ avec $X \supset\left\{x+y ; x \in S_{\varphi}\right.$, $\left.y \in B\left(0 ; r_{1}\right)\right\}$, et soit $N^{(i)}$ le noyau polypotentiel d'ordre $i(i=1, \cdots, p)$ dans $X$. Posons

$$
a_{m}=\int\left(N^{(p)}(x, y)-\sum_{i=1}^{p} N^{(p-i+1)} \varepsilon_{x, \boldsymbol{r}_{m}}^{(i)}(y)\right) d y,
$$

alors on a $a_{m}>0$ et

$$
\begin{aligned}
(-\Delta)^{p} \varphi(x) & =\lim _{m \rightarrow \infty} \frac{1}{a_{m}} \int(-\Delta)^{p} \varphi(y)\left(N^{(p)}(x, y)-\sum_{i=1}^{p} N^{(p-i+1)} \varepsilon_{x, \gamma_{m}}^{(i)}(y)\right) d y \\
& =\lim _{m \rightarrow \infty} \frac{1}{a_{m}}\left(\varphi(x)-\sum_{i=1}^{p}(-\Delta)^{i-1} \varphi(y) d \varepsilon_{x, \gamma_{m}}^{(i)}(y)\right) .
\end{aligned}
$$

Montrons la condition nécessaire. On prend une boule ouverte $B(x ; r)$ avec $\overline{B(x ; r)} \subset \Omega$. Soit $\delta$ un nombre $>0$ avec $\overline{B(x ; r+2 \rho)} \subset \Omega$. Alors, pour toute fonction $\varphi$ de classe $C^{\infty}$ à support compact dans $B\left(0 ; \frac{1}{2} \rho\right)$, on a $\Delta^{p} u^{\prime} * \varphi(x)=0$ dans un voisinage de $\overline{B(x ; r)}$, où $u^{\prime}$ est la restriction de $u$ sur $B(x ; r+\rho)$. Le support de $u^{\prime} * \varphi$ étant compact dans $\Omega$, on a

$$
u^{\prime} * \varphi(x)=N_{\Omega}^{(p)}(-\Delta)^{p} u^{\prime} * \varphi(x)
$$


dans $\Omega$. Ayant

$$
\left.N_{\Omega}^{(p)}(x, y)=\sum_{i=1}^{p} N_{\Omega}^{(p-i+1)} \boldsymbol{\varepsilon}_{x, r}^{(i)}(y) \text { dans } C \overline{B(x ; r}\right),
$$

on a

$$
u^{\prime} * \varphi(x)=\sum_{i=1}^{p} \int(-\Delta)^{i-1} u^{\prime} * \varphi(y) d \varepsilon_{x, r}^{(i)}(y)
$$

$\varphi$ étant arbitraire, on a

$$
u^{\prime}(x)=\sum_{i=1}^{p} \int(-\Delta)^{i-1} u^{\prime}(y) d \varepsilon_{x, r}^{(i)}(y),
$$

nous avons donc notre résultat. La démonstration est ainsi complète.

En explicitant les $\varepsilon_{x, r}^{(i)}$, on retrouve le théorème de valeur moyenne connu pour le fonction polyharmonique (cf. M. Nicolesco [3]). De la même manière, pour tout ouvert $\Omega_{0}$ dans $\Omega$, la fonction polyharmonique $u$ d'ordre $p$ dans $\Omega$ vérifie l'équation suivante: Pour tout $x$ de $\Omega_{0}$,

$$
u(x)=\sum_{i=1}^{p} \int(-\Delta)^{i-1} u(y) d \varepsilon_{x, C \Omega_{0}}^{(i)}(y)
$$

et par suite, $u$ est évidemment analytique dans $\Omega$.

Théorème 3. Soit $\Omega$ un ouvert borné de $R^{n}$, et soit $\left(f_{i}\right)_{i=1}^{p}$ un système de fonctions boréliennes et bornées sur $\Omega^{*}$. La fonction

$$
H_{\Omega}\left(x ;\left(f_{i}\right)_{i=1}^{p}\right)=\sum_{i=1}^{p} \int f_{i}(y) d \varepsilon_{x, C \Omega}^{(i)}(y)
$$

est polyharmonique d'ordre $p$ dans $\Omega .{ }^{1}{ }^{1}$

Démonstration. Soit $u_{i}(x)=\int f_{i}(y) d \varepsilon_{x, C \Omega}^{(i)}(y)(i=1, \cdots, p)$. D'après la proposition $2, u_{i}$ est analytique dans $\Omega$. On a

$$
\begin{aligned}
u_{i}(x) & =\iint f_{i}(y) d \varepsilon_{z, C \Omega}^{(1)}(y)\left(N^{(i-1)}(x, z)-\sum_{j=1}^{i-1} N^{(i-j)} \varepsilon_{x, C \Omega}^{(j)}(z)\right) d z \\
& =N^{(i-1)} u_{1}(x),
\end{aligned}
$$

et par suite,

1) $\left(\varepsilon_{x, C \Omega}^{(i)}\right)_{i=1}^{p}$ est le système balayé de $\left(\varepsilon_{x}, 0, \cdots, 0\right)$ sur $C \Omega$ relativement au système de noyaux polypotentiels dans un ouvert borné $X \supset \bar{\Omega}$. Il est indépendant de $X$ (cf. la proposition 3 ). 


$$
\Delta^{p} u_{i}(x)=(-1)^{i-1} \Delta^{(p-i+1)} u_{1}(x)=0
$$

dans $\Omega$, car $u_{1}$ est harmonique dans $\Omega$. La démonstration est complète.

Définition 1. La fonction $H_{\Omega}\left(x ;\left(f_{i}\right)_{i=1}^{p}\right)$ s'appelle la solution généralisée $d u$ problème de Riquier pour l'équation $\Delta^{p} u=0$ et pour la valeur frontière $\left(f_{i}\right)_{i=1}^{p}$.

Définition 2. On dit qu'un point $x_{0}$ de $\Omega^{*}$ est régulier d'ordre $p$ si l'on a, pour tout système $\left(f_{i}\right)_{i=1}^{p}$ de fonctions finies et continues sur $\Omega^{*}$,

$$
\lim _{\substack{x \rightarrow x_{0} \\ x \in \Omega}}(-\Delta)^{k-1} H_{\Omega}\left(x ;\left(f_{i}\right)_{i=1}^{p}\right)=f_{k}\left(x_{0}\right)(i=1, \cdots, p) .
$$

Examinons le point régulier d'ordre $p$ plus précisement. Le théorème typique est le suivant:

THÉORÈme 4. Soit $p \geqq 1$. Pour qu'un point $x_{0}$ de $\Omega^{*}$ soit régulier d'ordre $p$ il faut et il suffit que $x_{0}$ soit régulier au sens du problème de Dirichlet pour l'équation de Laplace.

Démonstration. Soit $x_{0}$ un point régulier au sens du problème de Dirichlet pour l'équation de Laplace, on a alors

$$
\lim _{\substack{x \rightarrow x_{0} \\ x \in \Omega}} \int f_{1}(y) d \varepsilon_{x, C \Omega}^{(1)}(y)=f_{1}\left(x_{0}\right)
$$

et pour $i>1$, on a

$$
\begin{aligned}
& \lim _{\substack{x \rightarrow x_{0} \\
x \in \Omega}}\left(-\Delta_{x}\right)^{i-1} \int f_{i}(y) d \varepsilon_{x, C \Omega}^{(i)}(y)=\lim _{\substack{x \rightarrow x_{0} \\
x \in \Omega}}\left(-\Delta_{x}\right)^{i-1} \iint f_{i}(y) d \varepsilon_{z, C \Omega}^{(1)}(y) N_{\Omega}^{(i-1)}(x, z) d z \\
& =\lim _{\substack{x \rightarrow x_{0} \\
x \in \Omega}} \int f_{i}(y) d \varepsilon_{x, C \Omega}^{(1)}(y)=f_{i}\left(x_{0}\right) .
\end{aligned}
$$

Ensuite, pour tout $x$ de $\Omega$ et pour tout $j \geqq 1$, on a

$$
\Delta^{i-1+j} \int f_{i}(y) d \varepsilon_{x, C \Omega}^{(i)}(y)=\Delta^{j} \int f_{i}(y) d \varepsilon_{x, C \Omega}^{(1)}(y)=0 .
$$

D'autre part, on a, pour tout $1 \leqq j \leqq i-1$,

$$
\begin{aligned}
& (-\Delta)^{i-1-j} \int f_{i}(y) d \varepsilon_{x, C \Omega}^{(i)}(y)=\int f_{i}(y) d \varepsilon_{z, C \Omega}^{(1)}(y) N_{\Omega}^{(j)}(x . z) d z \\
& =\int G_{\Omega}(x, w) N_{\Omega}^{(j-1)}(w, z) f_{i}(y) d \varepsilon_{z, C \Omega}^{(1)}(y) d z d w .
\end{aligned}
$$


En employant l'inégalité

$$
\sup _{x \in \Omega}\left|\iint N_{\Omega}^{(j-1)}(x, z) f_{i}(y) d \varepsilon_{z, C \Omega}^{(1)}(y) d z\right| \leqq\left(\sup _{x \in \Omega^{*}}\left|f_{i}(x)\right|\right)\left(\sup _{x \in \Omega} \int G_{\Omega}(x, z) d z\right)^{j-1},
$$

on obtient

$$
\lim _{\substack{x \rightarrow x_{0} \\ x \in \Omega}}(-\Delta)^{i-1-j} \int f_{i}(y) d \varepsilon_{x, C \Omega}^{(i)}(y)=0 .
$$

Par conséquent, on a, pour tout $k$,

$$
\lim _{\substack{x \rightarrow x_{0} \\ x \equiv \Omega}}(-\Delta)^{k-1} H_{\Omega}\left(x ;\left(f_{i}\right)_{i=1}^{p}\right)=f_{k}\left(x_{0}\right)
$$

L'inverse est évident, et par suite la démonstration est complète.

Finalement, nous voyons l'unicité de la solution du problème de Riquier.

Proposition 5. Soit $\Omega$ le même que ci-dessus. Si une fonction polyharmonique $u$ d'ordre $p$ et $\Delta^{i} u$ sont bornées dans $\Omega$ et admettent ses limites 0 quasi partout sur $\Omega^{*}$, on $a \quad u=0$.

En effet, soit $\left(\Omega_{m}\right)$ une suite croissante d'ouverts bornés avec $\bar{\Omega}_{m} \subset \Omega$ telle qu'on ait $\cup \Omega_{m}=\Omega$, alors on a, pour tout $x$ de $\Omega_{m}$.

$$
u(x)=\sum_{i=1}^{p} \int(-\Delta)^{i-1} u(y) d \varepsilon_{x, C \Omega_{m}}^{(i)}(y) .
$$

La suite $\left(\varepsilon_{x, C \Omega_{m}}^{(i)}\right)$ converge vaguement vers $\varepsilon_{x, C \Omega}^{(i)}$ avec $m \rightarrow \infty$, et par conséquent, faisant $m \rightarrow \infty$, on a $u(x)=0$ dans $\Omega$, puisque $u$ et $\Delta^{i} u$ sont bornées dans $\Omega$.

D'après ce théorème, on obtient immédiatement le corollaire suivant:

Corollaire 3. Soient $\Omega$ et $\left(f_{i}\right)_{i=1}^{p}$ les mêmes que ci-dessus. On a

$$
(-\Delta)^{k} H_{\Omega}\left(x ;\left(f_{i}\right)_{i=1}^{p}\right)=H_{\Omega}\left(x ;\left(f_{i}\right)_{i=k+1}^{p}\right) \text {. }
$$

Remarque. Nous pouvons donner les résultats à ceux-ci pour l'opérateur uniformement elliptique d'ordre 2 au lieu de $\Delta$. Pour cet opérateur, voir C. Stampacchia [5].

\section{RÉFÉRENCES}

[1] M. Brelot: Éléments de la théorie classique du potentiel, Les cours de Sorbonne 2eme édition, 1961. 
[2] M. Itô: Étude des fonctions polyharmoniques par la méthode du balayage, C.R. Acad. Paris, 267 (1968), 807-809.

[ 3 ] M. Nicolesco: Les fonctions polyharmoniques, Paris Hermann, 1936.

[4] M. Riesz: Intégrales de Riemann-Liouville et potentiels, Acta Sci. Math., Szeged, 9 (1938), 1-42.

[5] C. Stampacchia: Le problème de Dirichlet pour les équations elliptiques du second ordre à coefficients discontinues, Ann. Inst. Fourier, Grenoble, 15, 1 (1965), 189-256.

Faculté des Sciences d'Orsay, Orsay (France)

et Institut Mathématiuqe, Université de Nagoya 